\title{
Life Cycle Study of a Diabatic Rossby Wave as a Precursor to Rapid Cyclogenesis in the North Atlantic-Dynamics and Forecast Performance
}

\section{Journal Article}

Author(s):

Boettcher, Maxi; Wernli, Heini (D)

Publication date:

2011-06

\section{Permanent link:}

https://doi.org/10.3929/ethz-b-000037566

Rights / license:

In Copyright - Non-Commercial Use Permitted

Originally published in:

Monthly Weather Review 139(6), https://doi.org/10.1175/2011MWR3504.1 


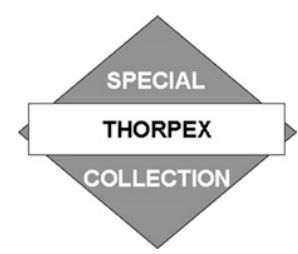

\title{
Life Cycle Study of a Diabatic Rossby Wave as a Precursor to Rapid Cyclogenesis in the North Atlantic-Dynamics and Forecast Performance
}

\author{
MAXI BOETTCHER \\ Institute for Atmospheric Physics, University of Mainz, Mainz, Germany \\ HEINI WERNLI \\ Institute for Atmospheric Physics, University of Mainz, Mainz, Germany, and Institute for \\ Atmospheric and Climate Science, ETH Zurich, Zurich, Switzerland
}

(Manuscript received 19 May 2010, in final form 27 January 2011)

\begin{abstract}
The life cycle of a North Atlantic cyclone in December 2005 that included a rapid propagation phase as a diabatic Rossby wave (DRW) is investigated by means of operational analyses and deterministic forecasts from the ECMWF. A quasigeostrophic omega diagnostic has been applied to assess the impact of upper-level forcing during the genesis, propagation, and intensification phase, respectively. The system was generated in the Gulf of Mexico as a mesoscale convective vortex (MCV) influenced by vertical motion forcing from a nearby upper-level trough. The DRW propagation phase was characterized by a shallow, low-level, diabatically produced potential vorticity (PV) anomaly that rapidly propagated along the southern border of an intense baroclinic zone. No significant upper-level forcing could be identified during this phase of the development. Eventually, explosive intensification occurred as the region of vertical motion forced by an approaching upper-level trough reached the position of the DRW. The rapid intensification of $34 \mathrm{hPa}$ in $24 \mathrm{~h}$ led to a mature extratropical cyclone in the central North Atlantic with marked frontal structures associated with a pronounced PV tower.

The performance of four operational deterministic ECMWF forecasts has been investigated for the DRW propagation and cyclone intensification. The forecasts showed a highly variable skill. Despite the fact that the DRW was initially well represented in all forecasts, two of them failed to capture the explosive intensification. By applying a DRW tracking tool, the low-level baroclinicity downstream of the DRW and the moisture supply to the south of the DRW could be identified as the key environmental parameters during DRW propagation. The subsequent cyclone intensification went wrong in two of the forecasts because of the missing interaction of the DRW and the upper-level trough. It is shown that this interaction can fail if the intensity of the DRW and/or the approaching upper-level wave are too weak, or in case of an erroneous structure of the upper-level trough leading to a phasing problem of the vertical interaction with the DRW. Therefore, the DRW intensification bears similar characteristics and forecast challenges as the extratropical reintensification of tropical cyclones.
\end{abstract}

\section{Introduction}

During the last few decades, the dynamics of explosively deepening extratropical cyclones has been a key subject of meteorological research. Prime examples for such explosive cyclone developments are the devastating "Presidents' Day cyclone" (Bosart 1981) and the "Queen

Corresponding author address: Maxi Boettcher, Institute for Atmospheric Physics, University of Mainz, Becherweg 21, D-55099 Mainz, Germany.

E-mail: maxi.boettcher@env.ethz.ch
Elizabeth II storm" (Gyakum 1983, 1991; Uccellini 1986). These investigations led to the discovery of a surface vortex existing prior to the onset of rapid intensification. Gyakum et al. (1992) generalized the hypothesis that antecedent vorticity development should be relevant to subsequent explosive development by looking into a large sample of cyclone events. They established the concept of a "two-phase development" for cyclones showing a pronounced near-surface vorticity precursor.

Simultaneously, theoretical studies using idealized twodimensional models investigated the impact of diabatic processes on a baroclinic unstable environment (Snyder 
and Lindzen 1991) and in mesoscale convective systems (Raymond and Jiang 1990). They identified a dynamicthermodynamic phenomenon, referred to as the diabatic Rossby wave (DRW), that generates potential vorticity (PV) disturbances at low levels diabatically in a similar manner as the meridional advection of PV in a classic Rossby wave. Parker and Thorpe (1995) studied DRWs situated over a frontal zone with a semigeostrophic model and described the basic mechanism of DRWs: advection of moist air masses on the eastern flank of the low-level vortex leads to upward motion, condensation, and diabatic heating. In this way, the localized low-level PV vortex is steadily renewed in the direction of the thermal wind by diabatic PV production.

Recently, some real case studies have been performed that associated the theoretically identified DRW phenomenon with the concept of low-level vortices acting as precursors for explosive cyclone intensification (Mallet et al. 1999; Wernli et al. 2002; Moore et al. 2008). The winter storm "Lothar" constitutes a clear example for a two-phase development. The precursor rapidly crossed the North Atlantic as a DRW before it intensified to one of the most harmful storms in central Europe in the last few decades. Wernli et al. (2002) identified an intensive straight zonal jet during the DRW propagation phase of Lothar far to the north of the low-level vortex and excluded a significant upper-level forcing of the surface low due to the absence of waves on the intense jet. They demonstrated that later in the development the DRW stimulated the formation of a small-scale upper-level trough, leading to the subsequent vertical interaction of the two PV anomalies. Unlike the bottom-up development of Lothar, Moore et al. (2008) investigated a cyclone near the U.S. east coast where the DRW intensified by interaction with an approaching (i.e., preexisting) upper-level trough. Both studies highlighted the key importance of moist processes for the DRW propagation by performing sensitivity experiments neglecting latent heat release (and surface fluxes in the later study). In these experiments the DRW mechanism came to a halt and the subsequent intensification was significantly weaker or even quasi-absent.

Moore and Montgomery $(2004,2005)$ conducted further idealized two- and three-dimensional model studies of DRWs. They suggested that regarding the environmental conditions, the baroclinicity and the low-level moisture supply are the most important parameters determining the size and amplitude of the propagating DRW. Considering real events of DRWs, their dynamics, their representation by operational models, and the key parameters for successful model predictions have not yet been investigated completely. Open questions concern the mechanisms that can lead to the generation of DRWs and the potential role of upper-level forcing during the DRW propagation. In addition, the results from the idealized investigations by Moore and Montgomery $(2004,2005)$ have not yet been verified in a more realistic model setting. And finally, it is unclear how well operational forecast models predict the generation, propagation, and intensification of a DRW. A first study dealing with this issue has been performed for the storm Lothar by Kenzelmann (2005). They analyzed the 50 simulations of the ECMWF ensemble prediction system and identified a strong sensitivity of the track and intensity of the mature storm to its structure and position relative to the jet axis during the DRW propagation phase. Detailed further studies are required for assessing the predictability of cyclones associated with DRWs. The present study aims at contributing some answers to these open questions.

The cyclone event investigated in this study occurred in December 2005 over the North Atlantic showing a typical two-phase development with a DRW precursor and an eventual explosive intensification. The study will investigate the processes during the genesis, propagation, and intensification of the cyclone, as well as the forecast performance of the deterministic ECMWF model for this particular event. In the next section the data and tools used in this study will be introduced. Section 3 contains a synoptic description of the cyclone life cycle and an analysis of the structure of the system for each development phase. With the aid of a quasigeostrophic vertical motion diagnostic the influence of the upperlevel forcing on the low-level system will be investigated. The forecast performance of this event will be explored by means of operational ECMWF forecasts with different lead times in section 4. The environmental conditions along the track of the DRW will be examined in the analysis and forecast data for investigating the different evolutions during both the propagation and intensification phase. Finally a summary and the conclusions will be presented in section 5 .

\section{Data and tools}

The data used in this study are operational analyses and forecasts from the European Centre for MediumRange Weather Forecasts (ECMWF). The fields with an original spectral resolution of T511L60 have been interpolated onto a regular grid with $0.6^{\circ}$ horizontal resolution on the original 60 vertical levels. The analyses and the forecasts are available every $6 \mathrm{~h}$. Four deterministic forecasts will be investigated that were started at 0000 and 1200 UTC on 17 and 18 December 2005, respectively. Further variables such as PV and the latent heating rate due to the condensation of water vapor have 
been derived from the ECMWF output data. The latent heating has been calculated with the method introduced by Berrisford (1988) and later used by Rossa et al. (2000) and Wernli et al. (2002). As a robust measure for lowlevel PV, PV has been interpolated from model levels to a stack of pressure levels between 975 and $800 \mathrm{hPa}$ with a separation of $25 \mathrm{hPa}$. The vertical average of PV on these eight pressure levels will be referred to as the "vertically averaged low-level PV."

As a special tool, the quasigeostrophic (QG) omega diagnostic described by Clough et al. (1996) has been applied. It allows calculating the height-attributable solution of the omega equation. Vertical motion on a specified level is separated into a contribution forced from upper levels and a contribution forced from lower levels. This enables us to distinguish ascent (and descent) induced by low-level flow features and upperlevel large-scale waves, respectively. Similarly to studies by Deveson et al. (2002) and Dacre and Gray (2009), the low-tropospheric layer has been specified from 1000 to $750 \mathrm{hPa}$. Differing from their studies the upper layer is specified here from 500 to $100 \mathrm{hPa}$ to exclude potential effects from low-level vortices extending into the middle troposphere. Vertical motion induced by these layers will be depicted at $700 \mathrm{hPa}$, which is typically close to the level of maximum vertical motion in extratropical cyclones. A few remarks should be made about the validity of using a QG diagnostic tool when investigating the dynamics of an intense and relatively small-scale diabatically produced DRW. First, the key aim of using this diagnostic in our study is to infer time periods when upper-level forcing for ascent is present in the vicinity of the DRW and time periods when this forcing is absent. Since the upper-level forcing is induced by synopticscale waves and jet stream circulations, the choice of a QG approach is justified. Second, the QG diagnostic strongly underestimates vertical motion in regions with intense latent heating. Therefore the QG low-level forcing for ascent is too weak in amplitude, but it correctly pinpoints the regions where the slantwise circulation associated with the low-level DRW leads to ascent and descent.

For investigating and comparing the DRW evolution in the analyses and forecasts, the tracking algorithm for DRWs developed by Kenzelmann (2005) has been refined. The algorithm searches in a smoothed version of the vertically averaged low-level PV field for grid points with PV maxima that exceed 0.8 potential vorticity units (PVU; $1 \mathrm{PVU} \equiv 1 \times 10^{-6} \mathrm{~K} \mathrm{~kg}^{-1} \mathrm{~m}^{2} \mathrm{~s}^{-1}$ ) and exhibit more than $50 \%$ relative humidity. For the horizontal smoothing, the low-level PV values have been averaged at every grid point with the eight horizontal neighbors. This reduces the sensitivity of the algorithm to very localized peaks in the PV distribution. Then, the DRW

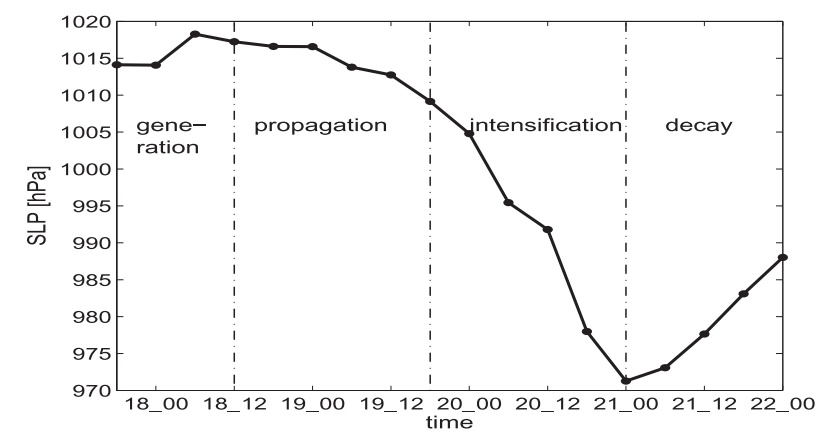

FIG. 1. Minimum SLP (hPa) time development of the DRW 17-22 Dec 2005 defining the development phases.

track is extended if at the next time step (i.e., $6 \mathrm{~h}$ later) a PV maximum exists within a box that reaches from the previous position $2^{\circ}$ to the west, $12^{\circ}$ to the east, $4^{\circ}$ to the north, and $2^{\circ}$ to the south. Additional criteria that need to be fulfilled at the first three consecutive time steps for classifying such a track as a propagating DRW are (i) the absence of stratospheric air at upper levels (i.e., PV should not exceed 2 PVU at any grid point in a box on $250 \mathrm{hPa}$ that extends $700 \mathrm{~km}$ to the west and north of the DRW); (ii) a significant downstream baroclinicity of $\Delta \theta \geq 5 \mathrm{~K}$ calculated by $\theta_{90}-\theta_{10}$, where $\theta_{90}$ corresponds to the 90th percentile of all $\theta$ values in a box that extends over about $800 \mathrm{~km}$ in the meridional direction (see section $4 \mathrm{~b}$ and gray box in Fig. 2d); and (iii) a propagation velocity of the low-level PV maximum of at least $3^{\circ}(6 \mathrm{~h})^{-1}$. To apply this algorithm the above-mentioned datasets have been interpolated onto equally spaced pressure levels (every $25 \mathrm{hPa}$ between 1000 and $100 \mathrm{hPa}$ ).

\section{Case overview based upon ECMWF analyses}

First, a general overview on the complete cyclone life cycle is given, based upon the time evolution of the cyclone's minimum sea level pressure (SLP) as shown in Fig. 1. The system originated from diabatic processes within a widespread convective precipitation area at about 1800 UTC 17 December (see below). The first period until 1200 UTC 18 December is hereafter referred to as the generation phase. Thereafter, the system associated with a SLP minimum of initially $1017 \mathrm{hPa}$ propagated rapidly toward the east without significant reduction of minimum SLP until 1800 UTC 19 December. These $30 \mathrm{~h}$ will be referred to as the propagation phase. The subsequent intensification phase features an explosive pressure deepening of almost $34 \mathrm{hPa}(24 \mathrm{~h})^{-1}$ and the cyclone reached its deepest core pressure of $971 \mathrm{hPa}$ at $0000 \mathrm{UTC}$ 21 December. Thereafter, the cyclone decayed slowly moving northeastward.

The following sections focus on the characteristics of the cyclonic system during the generation, propagation, 

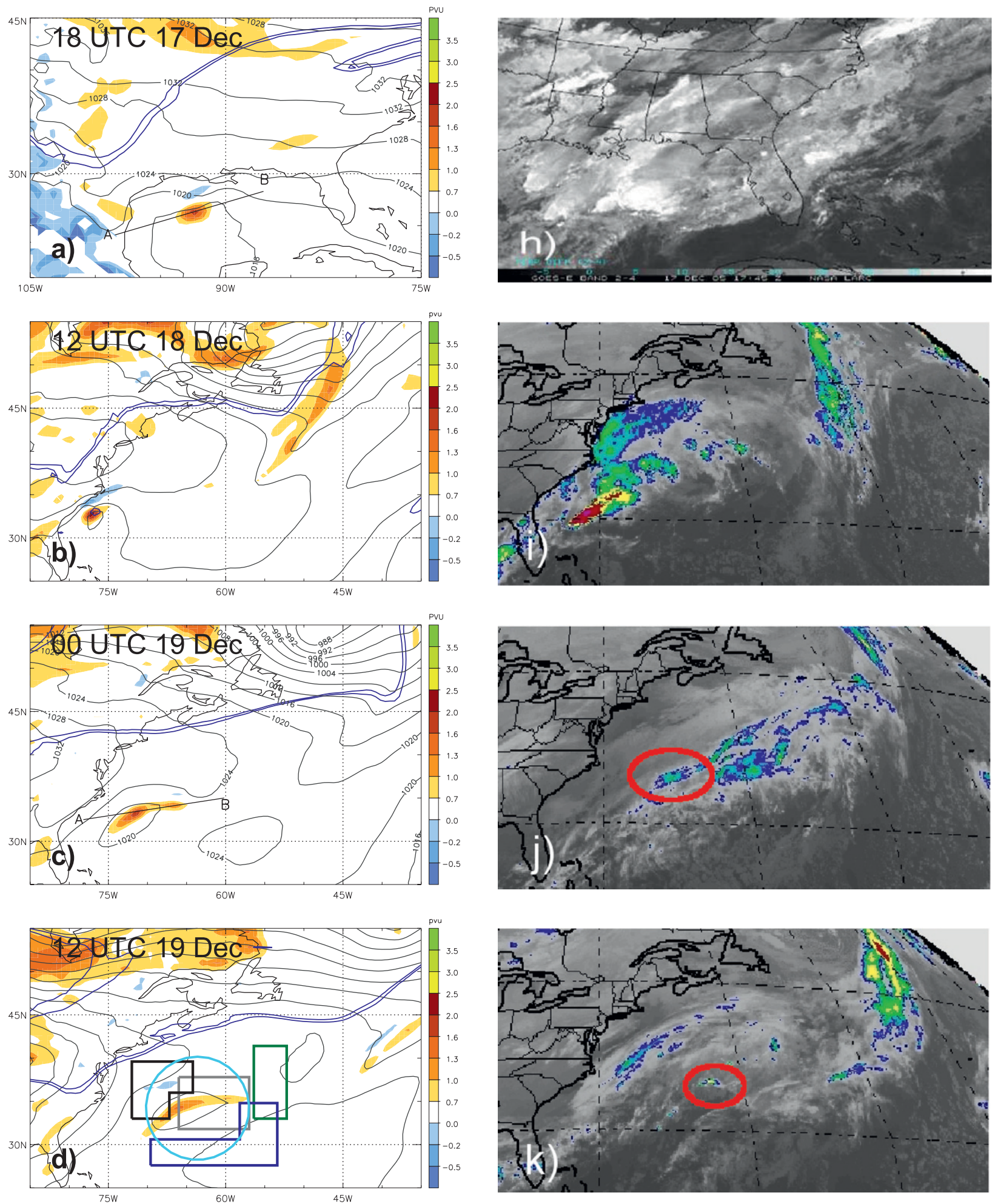

FIG. 2. (a)-(g) 975-800-hPa vertical averaged PV (colors, PVU), SLP (gray contours, interval 5 hPa), and PV contour (2 PVU at 315 K, blue). In (a), (c), and (g), line $\overline{\mathrm{AB}}$ marks the position of the cross section of Figs. 4b, 5b, and 6b. The positions of the boxes that are used for the quantitative forecast investigation in section $4 \mathrm{~b}$ are shown in (d). (h) IR satellite image GOES East (see online at http://angler.larc. nasa.gov). (i)-(m) IR satellite images GOES East (see online at http://squall.sfsu.edu, color shading in steps of $5^{\circ} \mathrm{C}$ from blue $-40^{\circ}$ to violet $-70^{\circ} \mathrm{C}$ ). (n) IR satellite image Meteosat (see online at http://www.sat.dundee.ac.uk).: (a),(h) 1800 UTC 17 Dec; (b),(i) 1200 UTC 18 Dec; (c),(j) 0000 UTC 19 Dec; (d),(k) 1200 UTC 19 Dec; (e),(l) 1800 UTC 19 Dec; (f),(m) 0600 UTC 20 Dec; and (g),(n) 0000 UTC 21 Dec 2005. 

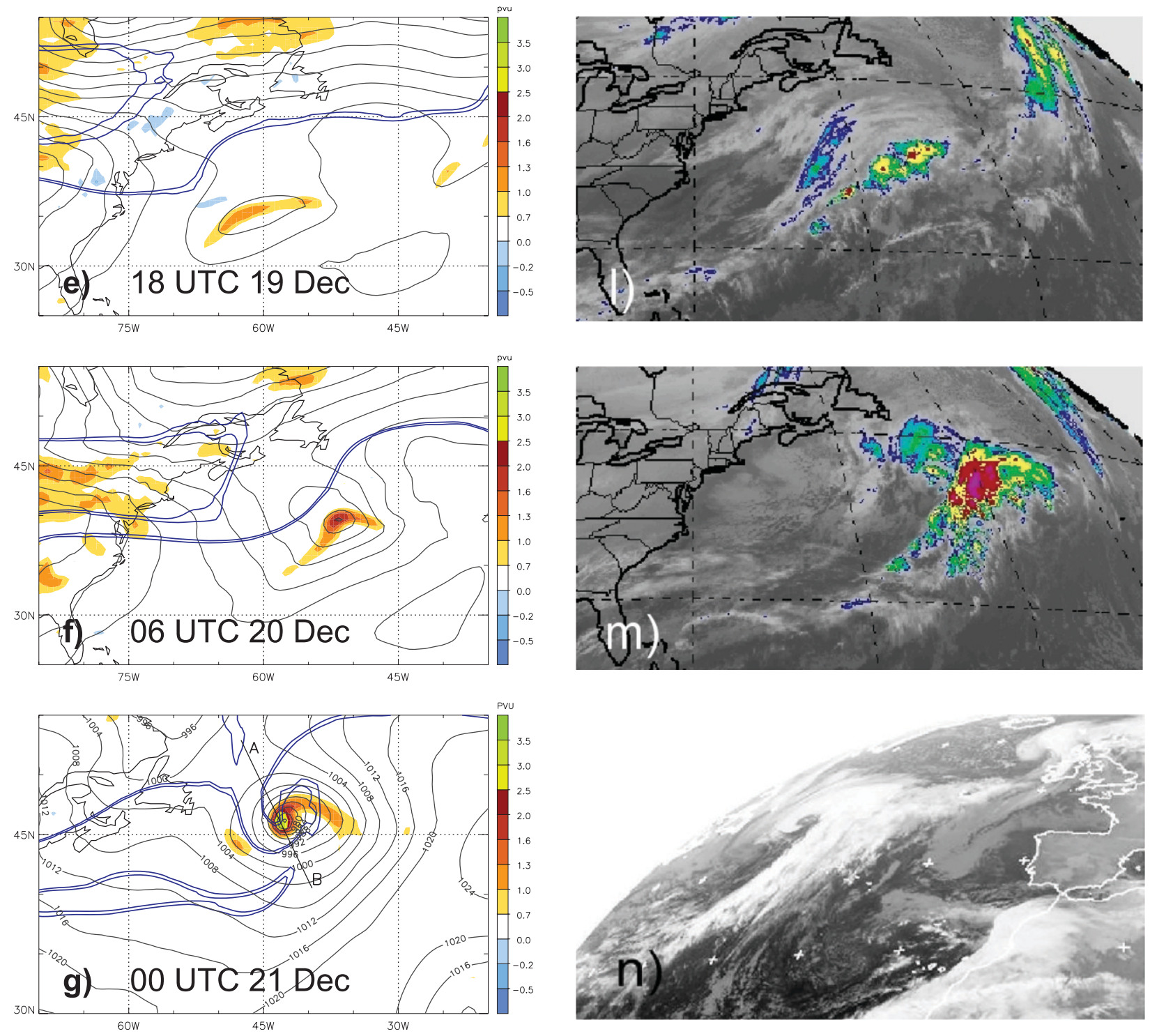

FIG. 2. (Continued)

and intensification phase, respectively. Specific time instants that are typical for each phase will be investigated in detail by analyzing the cyclone's vertical structure and the relative importance of low-level versus upper-level forcing of the vertical motion in the surrounding area of the system.

\section{a. Generation}

At 1800 UTC 17 December 2005 a positive low-level PV anomaly ${ }^{1}$ with an amplitude of about 2 PVU arises over the Gulf of Mexico, downstream of a small-scale

\footnotetext{
${ }^{1}$ The term "anomaly" is used to indicate significant deviations from a climatological value, which, in the lower extratropical troposphere is typically around 0.5 PVU.
}

upper-level trough located over the southwestern United States, according to the ECMWF analyses (Fig. 2a). A closed low-level circulation can be identified (not shown) that is accompanied by a shallow surface pressure minimum. The satellite image shows widespread stratiform clouds with embedded convective cells in the cyclone's genesis area (Fig. 2h). These features are characteristic for a mesoscale convective vortex (MCV). The associated precipitation region as observed by the Tropical Rainfall Measuring Mission (TRMM) satellite instrument covers a large proportion of the northern Gulf of Mexico (Fig. 3a). A tongue of low-level moisture of up to $14 \mathrm{~g} \mathrm{~kg}^{-1}$ (Fig. 3h) is advected toward the temperature gradient by southerly winds and will be essential for strong latent heating during the future evolution of the system. 

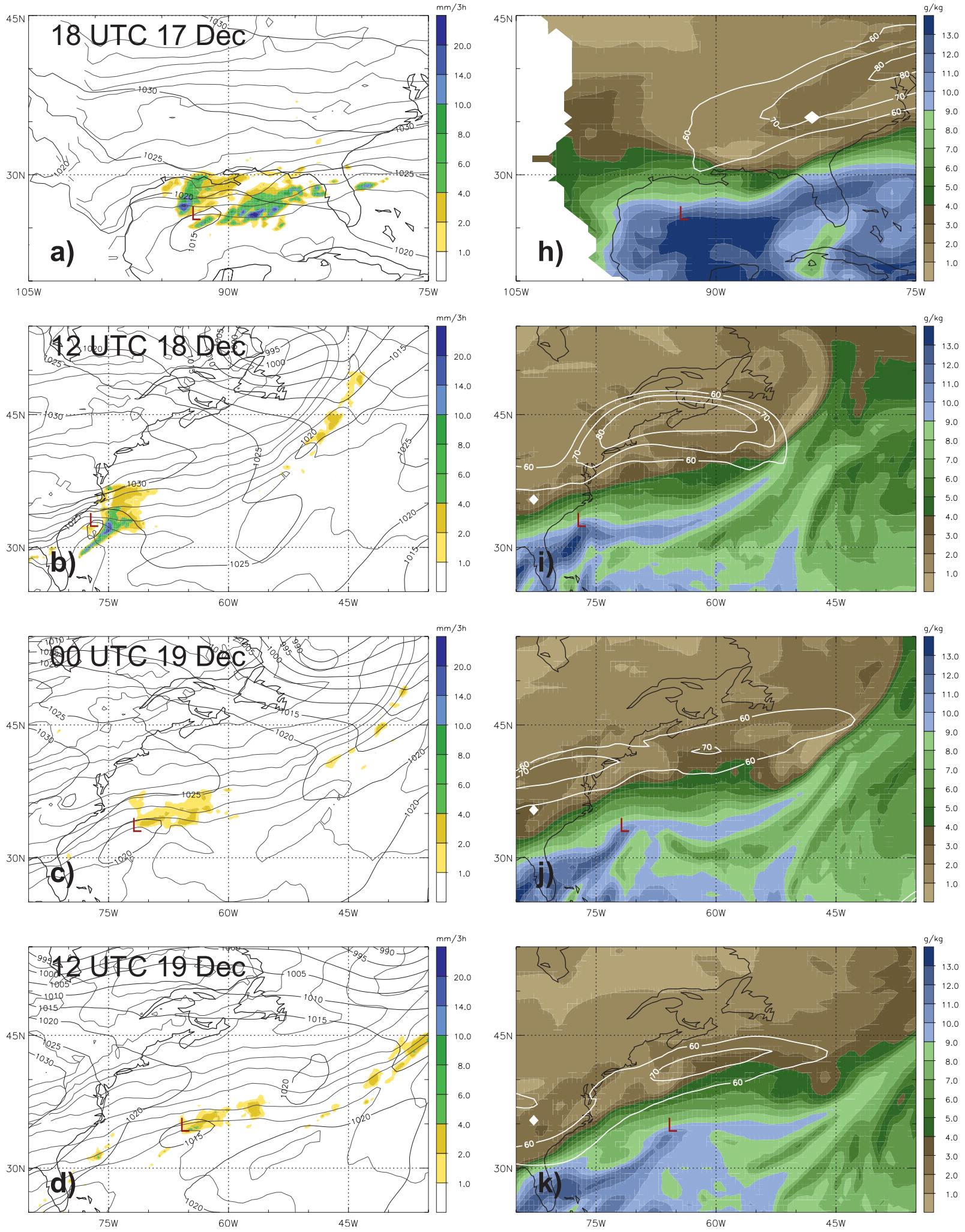

FIG. 3. (a)-(g) 3-hourly accumulated precipitation from TRMM satellite measurements [colors, $\mathrm{mm}(3 \mathrm{~h})^{-1}$ ] and potential temperature from ECMWF analyses at $900 \mathrm{hPa}$ (gray contours, interval $3 \mathrm{~K}$ ). (h)-(n) Specific humidity at $900 \mathrm{hPa}$ (colors, $\mathrm{g} \mathrm{kg}^{-1}$ ) and absolute wind at $250 \mathrm{hPa}$ (white contours, 60, 70, and $80 \mathrm{~m} \mathrm{~s}^{-1}$ ) at times as in Fig. 2. The red "L" marks the location of the SLP minimum. 

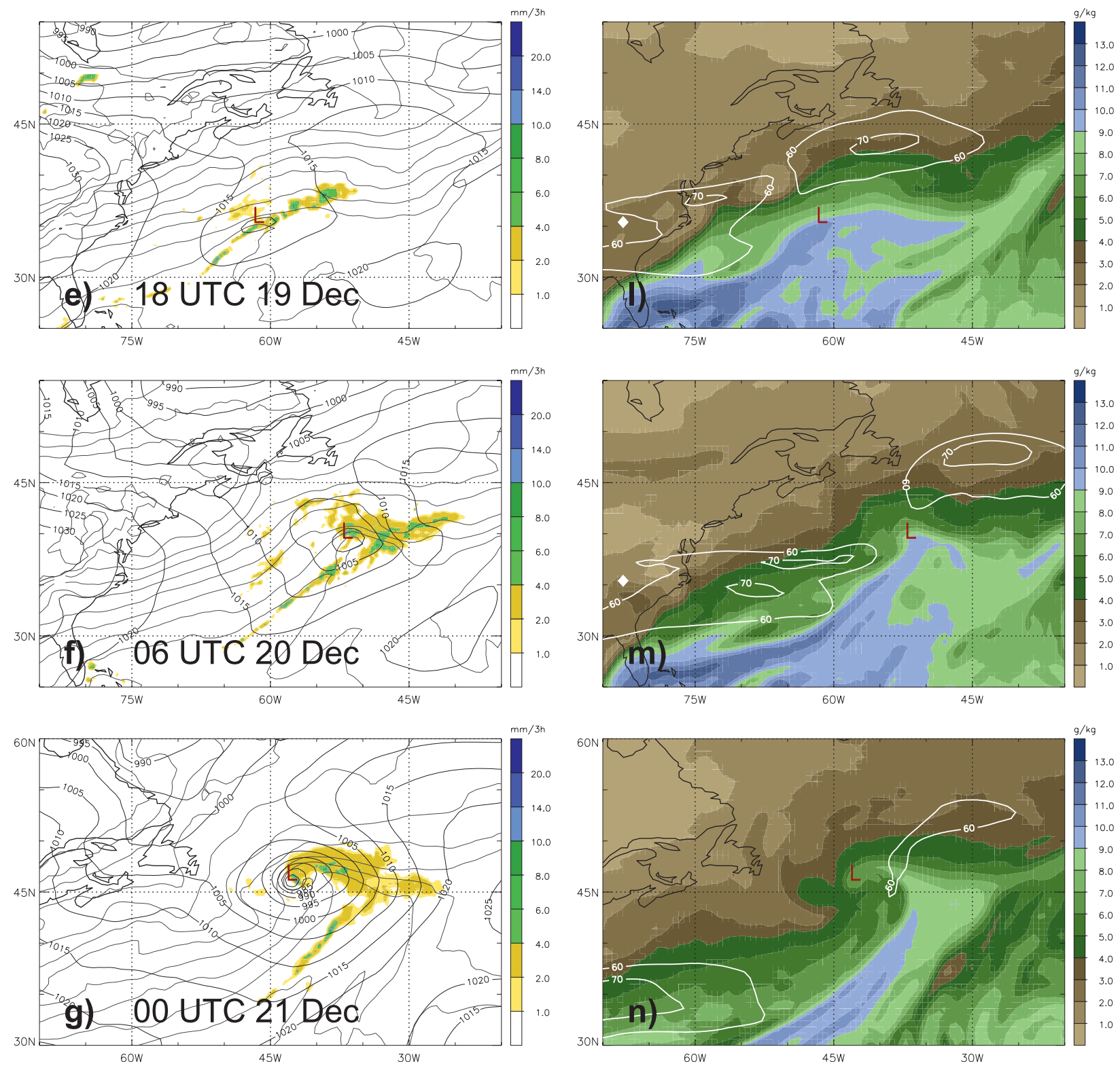

FIG. 3. (Continued)

At 1800 UTC 17 December, when the SLP minimum appears for the first time, weak ascent at $700 \mathrm{hPa}$ prevails over the Gulf of Mexico, apparently emanating from the upper-level trough located upstream (Figs. 2a and 4a). A small signature of ascent forced from low levels appears at the same time. The coherency of upper- and lower-level-induced vertical motion indicates their interaction. The low-level signature is a clear indication of a slantwise circulation in a baroclinic zone.

The vertical structure of the MCV shows maximum PV values of 2 PVU at about $900 \mathrm{hPa}$, and the vortex with anomalously large PV values ( $>1$ PVU) extends up to $600 \mathrm{hPa}$ (Fig. 4b). Latent heating occurs to the northeast of the positive low-level PV anomaly with maximum values of about $60 \mathrm{~K}(6 \mathrm{~h})^{-1}$ at $750 \mathrm{hPa}$. The green lines in Fig. $4 \mathrm{~b}$ denote the meridional wind component, which is strongest about $150 \mathrm{~km}$ to the east of the low-level PV maximum and that is responsible for the low-level ascent (and condensational heating) in the baroclinic zone downstream of the MCV. A few hours after its genesis, the system moves to the east and becomes separated from the upper-level trough. Eventually, the MCV crosses the Florida peninsula early on 18 December. At the same time the upper-level trough starts weakening. 

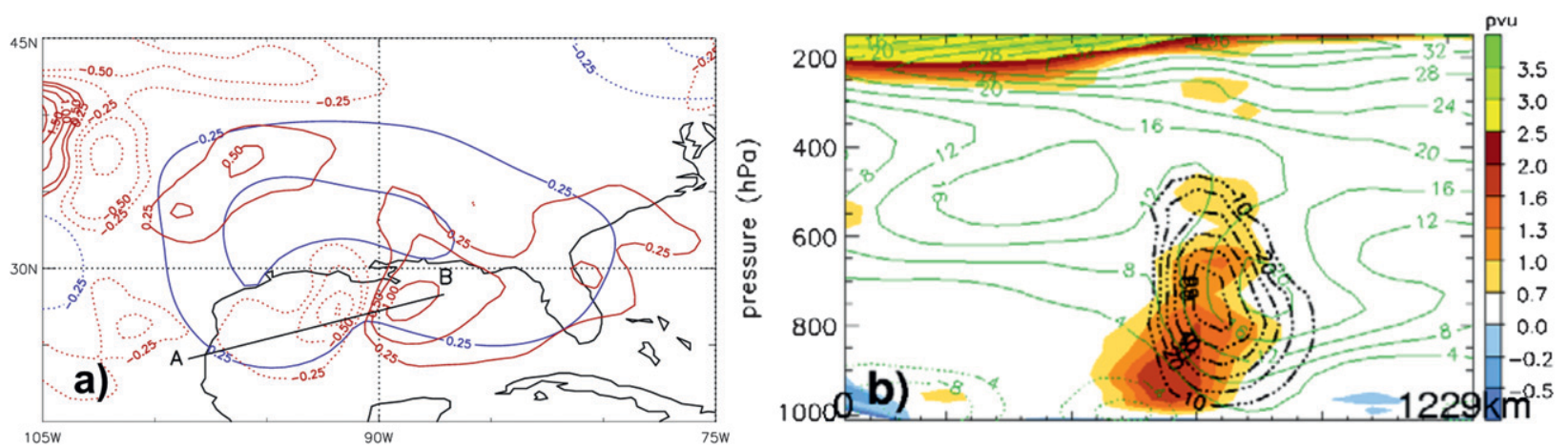

FIG. 4. MCV at 1800 UTC 17 Dec 2005 (a) QG vertical motion at $700 \mathrm{hPa}$ in $10^{-2} \mathrm{~m} \mathrm{~s}^{-1}$ (red contours: $\omega_{\mathrm{QG}}$ forced from between 1000 and $750 \mathrm{hPa}$, blue contours: forced from between 500 and $100 \mathrm{hPa}$; solid contours ascent, dashed contours descent). (b) Cross section through the line $\overline{\mathrm{AB}}$ as in Figs. 2a and 4a: PV (colors, PVU), meridional wind (green contours, interval $4 \mathrm{~m} \mathrm{~s}^{-1}$ ), and latent heating [black dashed contours, interval $10 \mathrm{~K}(6 \mathrm{~h})^{-1}$ ].

\section{b. Propagation}

After the low-level PV anomaly has entered the Atlantic basin at 1200 UTC 18 December, precipitation becomes more organized at the front side of the system (Figs. 2b and 3b). The cloud system of the former MCV spreads and stretches into a cloud band in the satellite image (Fig. 2i) along the baroclinic zone (Fig. 3b). The cloudiness in the area of the PV anomaly is not clearly separable from the clouds that are caused by the southerly flow around the subtropical high against the baroclinic zone (Figs. 2b,i). At 12 and 24 h later, a small-scale, vertically deep cloud embedded within the cloud band (marked by a red circle in Figs. 2j,k) can be associated with the low-level cyclonic system.

In the western North Atlantic downstream of the lowlevel PV anomaly the horizontal temperature gradient exceeds values of $10 \mathrm{~K}(500 \mathrm{~km})^{-1}$ (Figs. 3b,c,d). During the following propagation of the PV anomaly along the southern side of this intense baroclinic zone, the PV values decrease from 2.5 to 2 PVU (Figs. $2 b, c, d$ ) in harmony with decreasing precipitation amounts [from 25 to $8 \mathrm{~mm}(3 \mathrm{~h})^{-1}$ ] (Figs. 3b,c,d); that is, with a decrease of latent heating and associated diabatic PV production in the lower troposphere. The low-level PV anomaly propagates quickly to the east with a speed of about $13 \mathrm{~m} \mathrm{~s}^{-1}$. This is about twice the speed of the ambient wind at the height of the vortex in this weak pressure gradient environment.

The straight upper-level jet is about $900 \mathrm{~km}$ to the north of the low-level system at 0000 UTC 19 December (Figs. 2c and 3j). In agreement with this clear spatial separation between the low-level PV anomaly and the upper-level jet, no upper-level forced vertical motion can be identified in the vicinity of the low-level system (Fig. 5a). This corroborates the fact that no (significant) forcing from tropopause-level disturbances supports the propagation of the low-level PV anomaly. The absence of well-defined upper-level features and the fast propagation speed of the positive low-level PV anomaly along an intense baroclinic zone associated with strong moisture supply (Figs. 3i,j,k) and precipitation (Figs. 3b,c,d) indicate that during this period, the system corresponds to the concept of a DRW. ${ }^{2}$ Note as an aside that trajectory calculations (not shown) indicated that the tongue of high low-level moisture to the east of Florida (Fig. 3i) is associated with an intense event of tropical moisture export from the Gulf of Mexico (cf. Knippertz and Wernli 2010).

During the transition into a DRW, the upper-levelinduced vertical motion decreased gradually. However, the DRW itself causes a fairly intense low-level-induced horizontal dipole of vertical velocity with ascent ahead of the system and weaker descent behind. Looking at the vertical structure of the system during the propagation phase, ECMWF analyses show maximum PV values of about $2 \mathrm{PVU}$ at $900 \mathrm{hPa}$ in the center of the DRW (Fig. 5b). The strongest latent heat release occurs at the same height as for the MCV, but its intensity is weaker by about a factor of $2\left[30 \mathrm{~K}(6 \mathrm{~h})^{-1}\right]$. The vertical extent of the PV anomaly is smaller (up to 700 vs $600 \mathrm{hPa}$ for the MCV), and the induced meridional wind speed with a maximum of about $12 \mathrm{~m} \mathrm{~s}^{-1}$ is weaker. A major difference during the DRW propagation phase and the previous generation phase as a $\mathrm{MCV}$ is the maintenance (and propagation) of the DRW without vertical motion forcing from the upper troposphere. During its propagation phase, the DRW can thus

\footnotetext{
${ }^{2}$ Although the system shows a localized vortexlike circulation (and not a wavelike vorticity structure), we prefer to refer to this system as a DRW (instead of a diabatic Rossby vortex, cf. Moore and Montgomery 2004) in order to highlight the wavelike propagation mechanism (as opposed to the advection of a coherent vortex).
} 

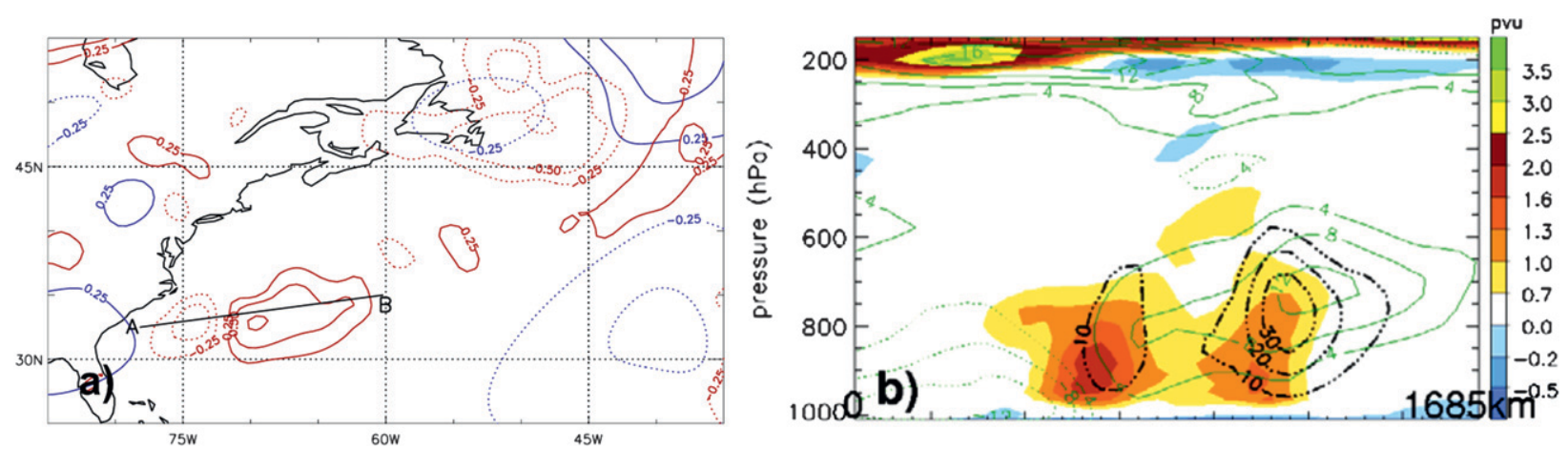

FIG. 5. As in Fig. 4, but the position of the cross section through the line $\overline{\mathrm{AB}}$ is as in Figs. 2c and 5a. Here at 0000 UTC 19 Dec 2005.

be considered as a shallow, diabatically driven low-level cyclonic system.

\section{c. Intensification}

Starting at 1800 UTC 19 December, the cloud structure associated with the DRW evolved into a leaf cloud (Figs. 21,m) indicating the start of a strong cyclone intensification. A distinct drop of the central SLP value (Fig. 1), the development of fronts, and this structural change in the satellite imagery indicate the transition of the DRW into an intense extratropical cyclone. The transition is accompanied by an increase in size from the small-scale shallow surface cyclone associated with the DRW to a synoptic-scale extratropical cyclone with a horizontal extent of about $1500 \mathrm{~km}$.

The transition of the DRW into an extratropical cyclone with typical frontal features occurs when the low-level DRW starts to interact with an approaching upper-tropospheric wave (Figs. 2e,f). This interaction can be identified as a vertical superposition of ascent induced by the DRW at lower levels and by the approaching trough at upper levels (Fig. 6a). Precipitation associated with the system increases again, in particular along the well-defined surface fronts (Figs. 3e,f). The positive lowlevel PV anomaly is now located in the equatorward entrance region of the upper-level jet (Figs. 31,m).
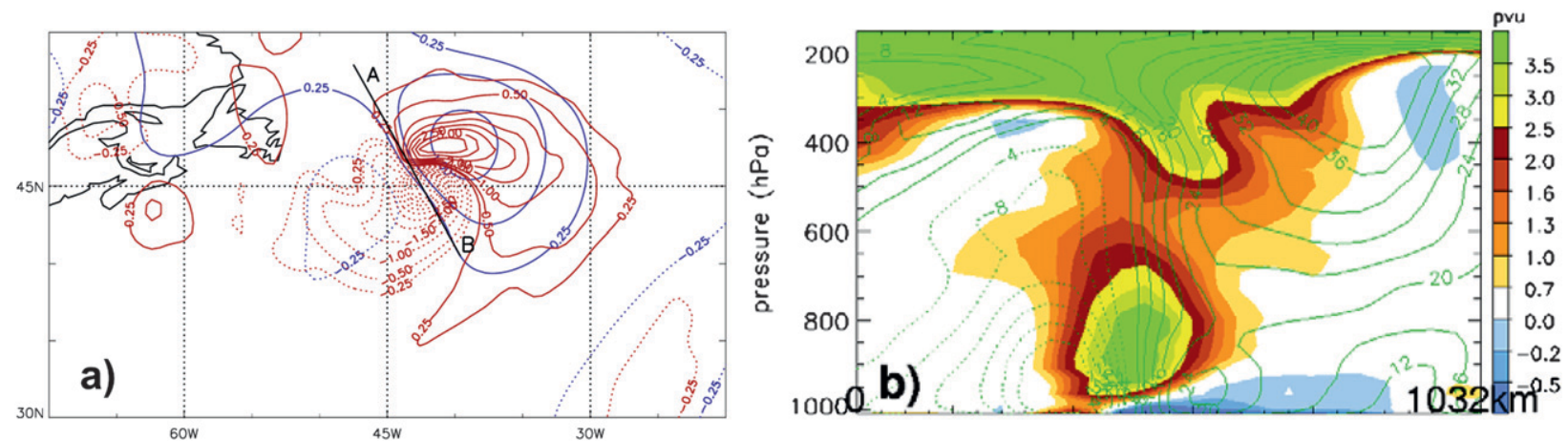

FIG. 6. As in Fig. 4, but the position of the cross section through the line $\overline{\mathrm{AB}}$ is as in Figs. $2 \mathrm{~g}$ and $6 \mathrm{a}$. Here at $0000 \mathrm{UTC} 21 \mathrm{Dec} 2005$. 

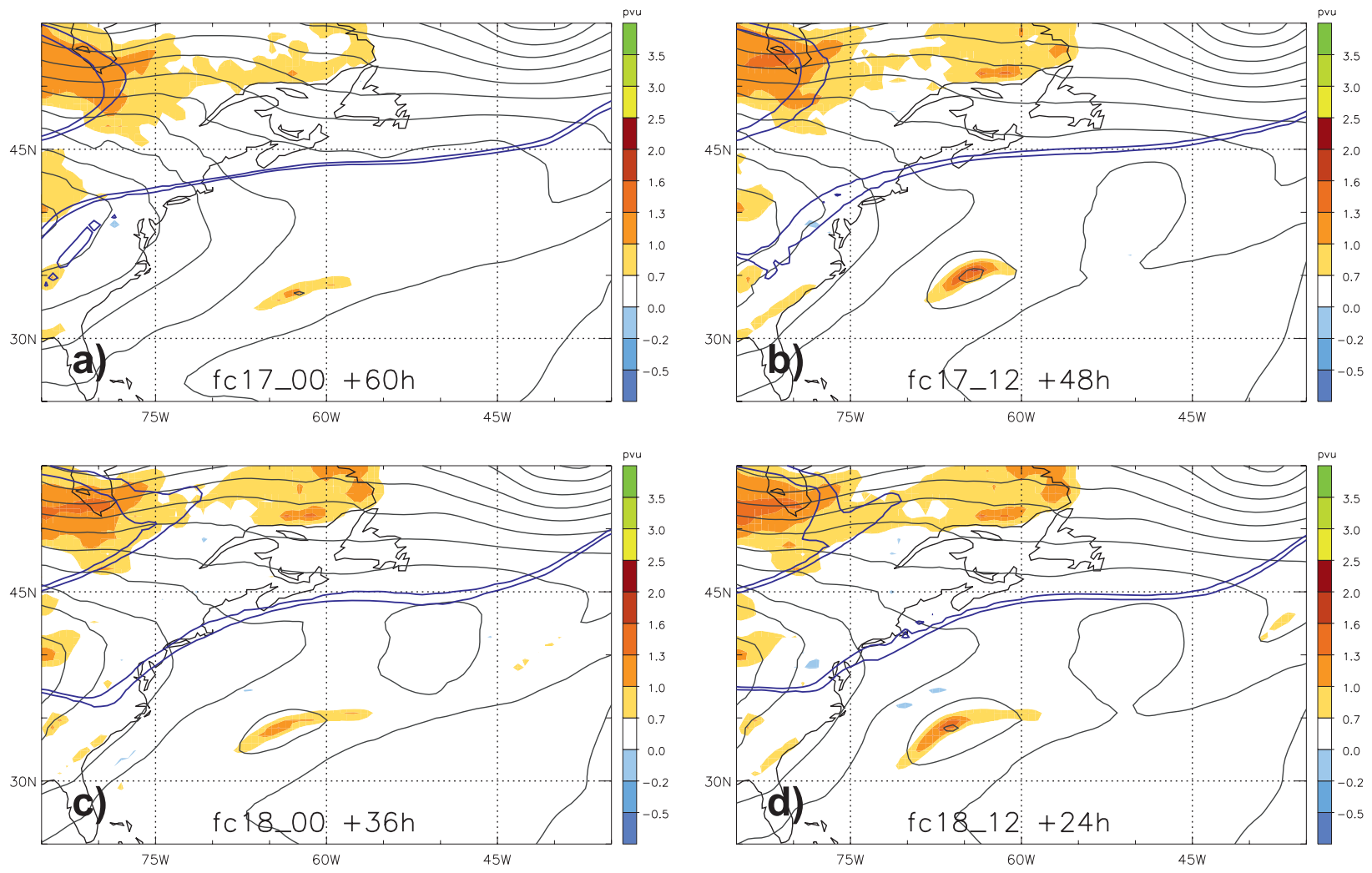

FIG. 7. Low-level averaged PV $975-800 \mathrm{hPa}$ (colors, PVU), SLP (gray contours, interval $4 \mathrm{hPa}$ ), and 2 PVU at $315 \mathrm{~K}$ (blue contour) for the investigated forecasts at 1200 UTC 19 Dec 2005.

infrared satellite image the mature stage of the cyclone is associated with a major cloud head enclosing a distinct dry slot in the center (Fig. 2n). Clouds extend over the warm front, which corresponds to the most active moistdiabatic region of the cyclone at this stage of the development.

Thereafter, the intensity of the cyclone decreases slowly on a northeasterly track. On 23 December the surface cyclone dissipates to the south of Iceland about 6 days after its genesis.

\section{Investigation of operational ECMWF forecasts}

In this section a set of operational deterministic ECMWF forecasts (with differing lead times) will be considered in order to (i) analyze the robustness and quality of the prediction of this rapidly propagating and explosively intensifying DRW, and (ii) to further investigate the dynamical and physical processes at play during the propagation and intensification phases. The forecasts that will be investigated were initiated at 0000 and 1200 UTC on 17 and 18 December 2005, respectively, that is $0-36 \mathrm{~h}$ prior to the beginning of the propagation phase of the DRW (see Fig. 1). In the following, these forecasts will be referred to as fc17_00, fc17_12, fc18_00, and fc18_12.

\section{a. General overview}

The DRW is correctly generated by the forecasts fc17_00 and fc17_12 at 1800 UTC 17 December, whereas the low-level PV anomaly to the east of Florida is already present in the initial conditions of fc18_00 and fc18_12 (not shown). At the initial time of the DRW propagation phase (1200 UTC 18 December), the strength and position of the DRW is very similar in all forecasts and the analysis (not shown). The differences between the forecasts grow substantially during the next few hours. At 1200 UTC 19 December (i.e., toward the end of the propagation phase), the DRW is weakest and no longer associated with a closed SLP contour in the forecast fc17_00 (Fig. 7a). The strongest DRW with the highest low-level PV values is produced in the forecast fc17_12 (Fig. 7b). The DRWs in the later forecasts are intermediate in intensity. In addition to the intensity differences, small position differences appear. The weakest DRW in fc17_00 is located slightly farther to the south and the slowest propagating DRW in fc18_12 is closest to the development in the analyses (cf. Fig. 2d). The tropopause 

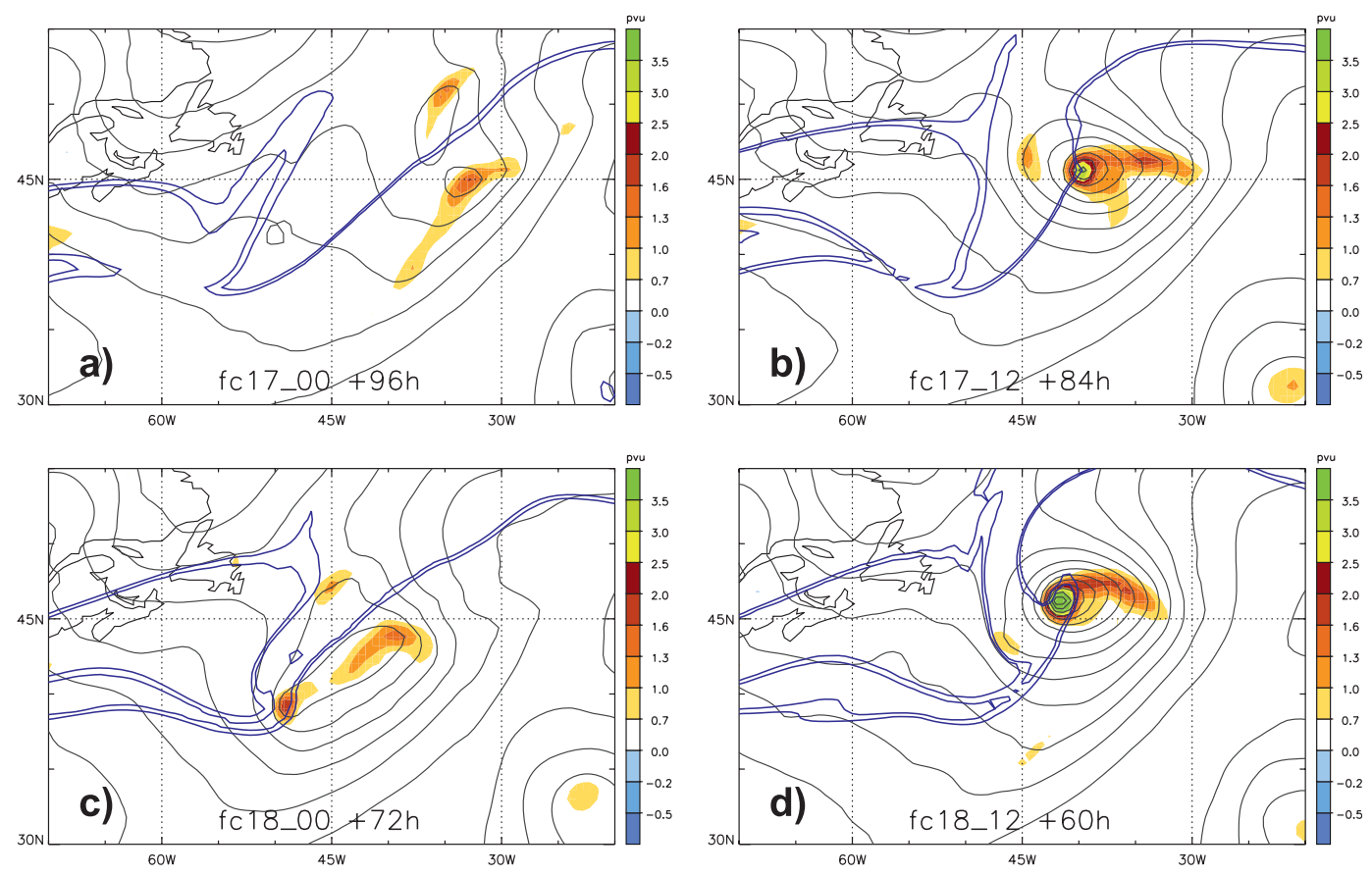

FIG. 8. As in Fig. 7, but at 0000 UTC 21 Dec 2005.

contour on $315 \mathrm{~K}$ (blue lines in Fig. 7) differs slightly between the forecasts, which does not yet have a direct influence on the low-level system at this time.

At 0000 UTC 20 December [i.e., at the beginning of the intensification phase (not shown)], the weakest DRW in fc17_00 exhibits the shortest distance to the approaching trough from the west. The other (later) forecasts represent the waves at the tropopause with a slightly stronger amplitude. The intense DRWs in fc17_12 and fc18_12 already show an elongated bended shape that indicate the genesis of warm and cold fronts and a distinct SLP minimum. These two systems are located farther north than the forecasts producing a weaker SLP minimum around the less intense DRWs.

During the next $24 \mathrm{~h}$ a strong deepening of the cyclone's minimum SLP comparable to that in the analyses only occurred in the forecasts fc17_12 and fc18_12 (Fig. 8). In fc17_12 the minimum SLP decreased during this time period from 1005 to $979 \mathrm{hPa}$, and in fc18_12 from 1003 to $964 \mathrm{hPa}$. At 0000 UTC 21 December (and thereafter), the cyclones in these forecasts are located below the low tropopause and they reveal strong SLP gradients and high low-level PV values in their center (Figs. 8b,d). In contrast, the forecasts fc17_00 and fc18_00 failed in capturing the cyclone intensification (Figs. 8a,c). Their 24-h decrease of minimum SLP only amounts to 9 and $11 \mathrm{hPa}$, respectively. These weak cyclones are still located on the warmer side of the upper-level jet. Forecast fc18_00 shows some pressure deepening and a second PV maximum in the elongated cyclone center. The more eastern PV maximum is associated with the former DRW. Compared to $24 \mathrm{~h}$ earlier, its amplitude has slightly decreased. This feature will be described in more detail at the end of section 4 .

Later, also the previously weak cyclones intensify. However, the processes leading to the intensification differs from the development revealed by the analyses. The cyclone in fc17_00 undergoes a sudden pressure deepening after 1200 UTC 21 December resulting in a SLP minimum of $982 \mathrm{hPa}$ at 0000 UTC 22 December about $700 \mathrm{~km}$ to the south of Iceland (not shown). The cyclone in fc18_00 intensifies steadily and reaches a SLP minimum of $970 \mathrm{hPa}$ at 1200 UTC 23 December near the Greenland east coast. In contrast, the cyclones in the successful forecasts weaken slowly as they pass to the south of Iceland and move toward Scandinavia (Fig. $9 \mathrm{~b}, \mathrm{~d})$. In summary, the forecasts fc17_00 and fc18_00 do not accurately represent the intensity, timing, and location of the deepening of the DRW into an intense extratropical cyclone.

Two of the four investigated forecasts showed development scenarios that severely differ from the analyses, already during the propagation phase and even more pronounced during the intensification phase. This calls for a closer examination that aims to reveal the reasons for the differing life cycles. In the following section the DRW tracking algorithm (see section 2) will be applied to quantitatively investigate key variables of 

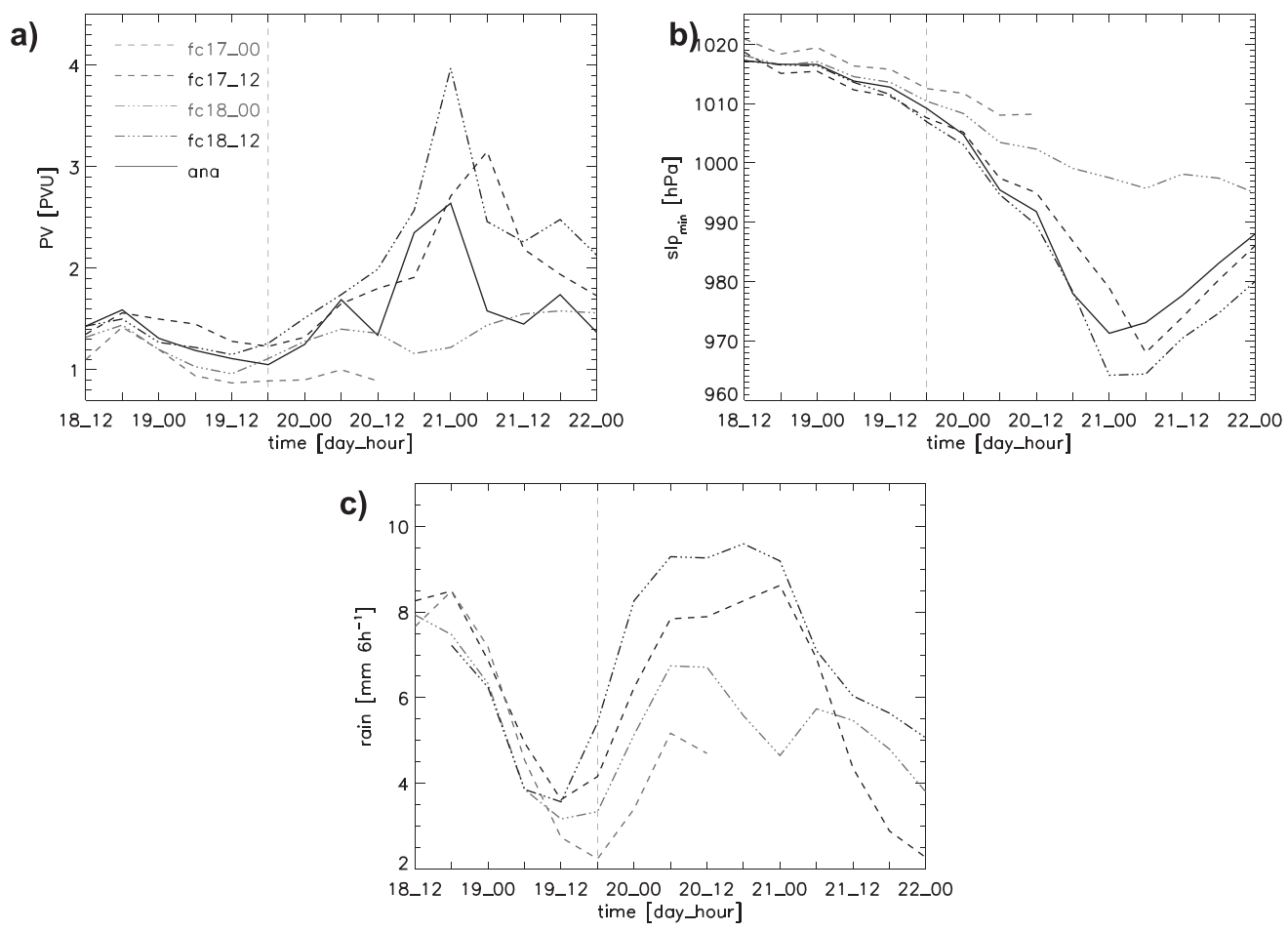

d)
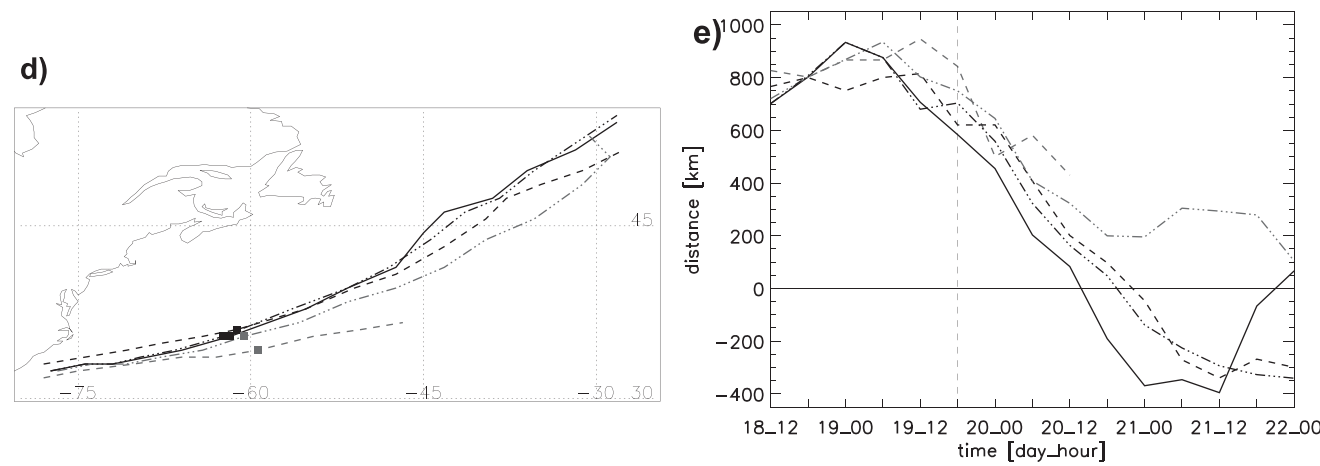

FIG. 9. Time development of (a) 975-800-hPa vertical and horizontal averaged PV (PVU), (b) minimum SLP $(\mathrm{hPa}),(\mathrm{c})$ averaged precipitation in the light blue circle in Fig. $2 \mathrm{~d}\left[\mathrm{~mm}(6 \mathrm{~h})^{-1}\right],(\mathrm{d})$ track (the squares mark the transition from the propagation to the intensification phase), and (e) distance to the upper-level jet for the forecasts and the analysis for the time period 1200 UTC 18 Dec-0000 UTC 22 Dec 2005. The dashed-dotted line separates the propagation from the intensification phase.

the system and the environmental conditions along the track.

\section{b. Quantitative investigation}

\section{1) INTENSITY AND TRACK OF THE DRW}

During the propagation phase, the amplitude of the low-level PV is considered as an indicator for the strength of the DRW. Figure 9a shows the time development of the smoothed maximum low-level PV. At the start of the DRW propagation at 1200 UTC 18 December the maximum PV values in the forecasts differ between 1.1 and 1.4 PVU. Except for the first $6 \mathrm{~h}$, the low-level PV values generally decrease slowly in all forecasts and the analyses until the end of the propagation phase at 1800 UTC 19 December (highlighted by the dashed-dotted vertical line in Fig. 9). The strongest DRW occurs in the forecast fc17_12 throughout the propagation. At 1800 UTC 19 December maximum low-level PV values vary between 0.9 PVU in fc17_00 and around 1.25 PVU in the forecasts started at 1200 UTC. At this time, the DRW in the analysis is also relatively weak (1.0 PVU) and compares best with the forecast fc18_00. 
During the intensification phase, after 1200 UTC 20 December, maximum PV values rise dramatically in the analyses and the two forecasts initiated at 1200 UTC. In these forecasts smoothed maximum low-level PV reaches up to 4 and 3.2 PVU, exceeding the peak value in the analysis (2.4 PVU). The low-level PV in fc18_00, however, evolves without considerable intensification as the maximum does not exceed 1.6 PVU (which is still an anomalously large low-level PV value). The DRW in fc17_00 becomes too weak to be traceable with values below 0.8 PVU after 1200 UTC 20 December.

The time evolution of the SLP minimum associated with the system (Fig. 9b) corresponds to the minimum grid point value in the area surrounding the center of the PV anomaly. Initially, the DRW is accompanied by a shallow SLP minimum between 1021 and $1018 \mathrm{hPa}$. Then during DRW propagation, SLP decreases steadily in all forecasts. The forecasts that capture the intensification generate an explosive pressure deepening that slightly exceeds the deepening rate in the analyses in case of fc18_12 by $4 \mathrm{hPa}(24 \mathrm{~h})^{-1}$. Compared to the analyses, the cyclone in this forecast captures the time of minimum SLP but overestimates its intensity by $5 \mathrm{hPa}$. The forecast fc17_12 better represents the SLP minimum but has a weak timing error of $6 \mathrm{~h}$.

The time evolution of precipitation averaged near the cyclone center (see the light blue circle in Fig. 2d, which is moving with the system) is in qualitative agreement with the time evolution of the diabatically produced low-level PV (Figs. 9a,c). Consistently, PV values and precipitation decrease in a similar manner during the propagation phase revealing a weakening of moist condensational activity. ${ }^{3}$ Precipitation is most intense for the strongest DRW. The diabatic contribution to the explosive intensification in the analyses and the forecasts fc17_12 and fc18_12 shows up as a strong increase of precipitation in the vicinity of the cyclone center after 1800 UTC 19 December. In contrast, for the erroneously weak developments in fc17_00 and fc18_00 the precipitation increase is smaller by a factor of about 2 compared to the strongly deepening scenarios.

The track of the system corresponds to the time sequence of the position of the center of the low-level PV anomaly (Fig. 9d). The tracks start with only a small position difference at 1200 UTC 18 December. During the propagation phase, the northernmost DRW track occurs in fc17_12. At the end of the propagation phase (highlighted with square symbols in Fig. 9d) the DRW is

\footnotetext{
${ }^{3}$ Note that we cannot expect a perfect correlation between the two quantities, since precipitation is at best an integral measure of diabatic heating whereas the diabatic PV production depends strongly on the vertical gradient of the latent heating.
}

located about $200 \mathrm{~km}$ too far to the south in the forecast fc17_00. Subsequently, the explosively deepening systems in fc17_12 and fc18_12 follow a more northerly track, in agreement with the analysis track. At the end of the investigated time period, at 0000 UTC 22 December, the cyclone positions vary only slightly in longitude but rather drastically, by about $600 \mathrm{~km}$, in latitude. For the system in fc18_12 (i.e., for the most intense cyclone), the agreement with the analyses is best both in terms of position and intensity. The tracks of the systems that strongly underestimated intensification are too far to the south. The differing meridional motion of the systems prompts the following analysis of their distance to the upper-level jet stream.

The distance of the DRW to the upper-level jet axis is determined as the horizontal distance from the DRW center to the nearest grid point with at least 2 PVU on $250 \mathrm{hPa}$. The sign convention is such that positive (negative) values correspond to the DRW being south (north) of the jet axis. During the propagation phase, the DRW is located more than $600 \mathrm{~km}$ to the south of the upperlevel jet, as shown in Fig. 9e. The intensification of the cyclone coincides with its approach to the upper-level jet axis. Until 0600 UTC 20 December, the distance to the jet is very similar for all forecasts (and slightly larger than in the analyses). Thereafter, the evolutions differ and the DRWs in the forecasts with explosive intensification continue to approach the jet until they cross it at 0000 UTC 21 December, which is $6 \mathrm{~h}$ later than in the analyses. Only if the cyclones cross the jet axis, they can establish a vertically coherent PV tower, which constitutes the most favorable superposition of low- and upper-level PV anomalies and their associated cyclonic circulations. The DRW in fc17_00 crosses the jet axis $12 \mathrm{~h}$ later and, consistently, intensifies much too late (see Fig. 9b). In the forecast fc18_00, the DRW comes never closer than about $200 \mathrm{~km}$ to the jet, again in qualitative agreement with the time evolution of minimum SLP that indicates no significant intensification.

\section{2) ENVIRONMENTAL CONDITIONS DURING DRW PROPAGATION}

The tracking algorithm also provides the opportunity to measure physical and dynamical parameters in the vicinity of the DRW in predefined moving boxes (i.e., in boxes with a fixed position relative to the center of the propagating low-level PV vortex). The position and the size of the boxes are shown in Fig. $2 d$ and have been set empirically based upon the following rationale. The variables of interest (which are thought to potentially influence the propagation and intensification of the DRW) 

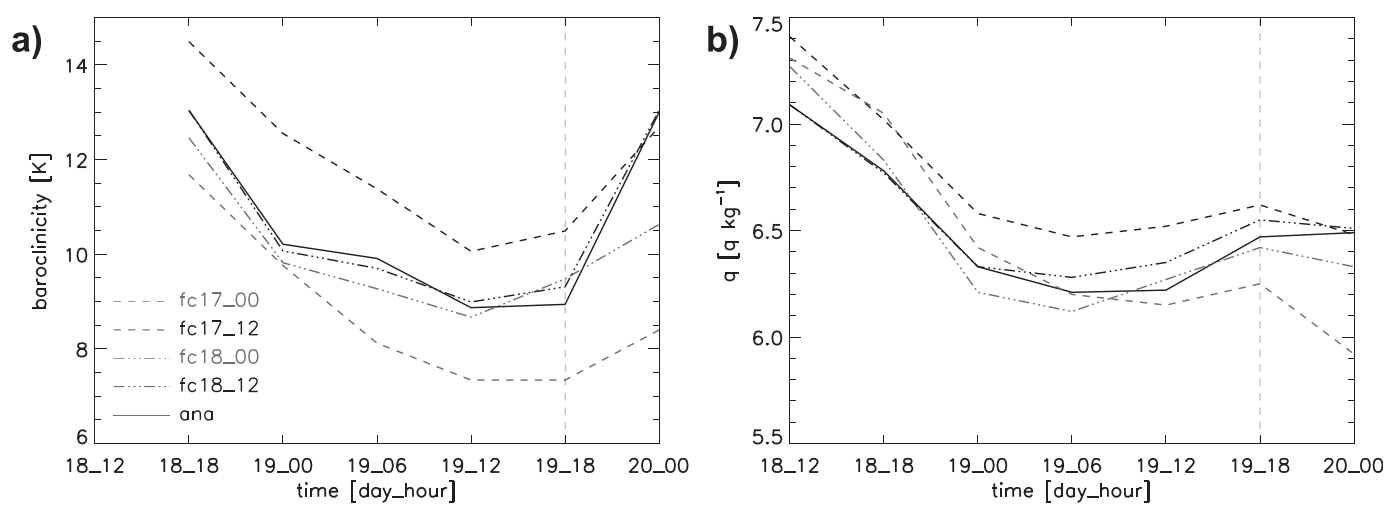

FIG. 10. Time development of (a) baroclinicity at $950 \mathrm{hPa}\left(T_{10 \% \max }-T_{10 \% \min }\right.$ in the green box in Fig. $\left.2 \mathrm{~d}, \mathrm{~K}\right)$, and (b) specific humidity (averaged over 1000 and $700 \mathrm{hPa}$ in the blue box in Fig. 2d, $\mathrm{g} \mathrm{kg}^{-1}$ ) during DRW propagation during 1200 UTC 18 Dec-0000 UTC 20 Dec 2005. The dashed-dotted line separates the propagation from the intensification phase.

are low-level baroclinicity and moisture, upper-level PV, and midtropospheric vertical velocity. ${ }^{4}$

Low-level baroclinicity is evaluated far downstream (i.e., about $800 \mathrm{~km}$ to the east) of the actual position of the DRW in order to capture the environmental temperature gradient before it is distorted by the approaching DRW. It is calculated as the difference between the 90th and 10th percentile of the gridpoint values of potential temperature at $950 \mathrm{hPa}$ within the green box shown in Fig. 2d. In addition, when displaying this parameter in Fig. 10a, the values are shifted $6 \mathrm{~h}$ in time in order to approximately compensate for the time it takes the DRW to reach this box. In all forecasts and the analyses, this quantity decreases along the track of the DRW (Fig. 10a). Considering the (idealized) DRW propagation mechanism, this continuously reduced baroclinicity (which is associated with a decrease of the meridional slope of the isentropes; not shown) might lead to a continuously weaker ascent along the sloping isentropes induced by the circulation of the DRW resulting in reduced latent heating and diabatic PV production. This is qualitatively confirmed by the fact that the most intense DRW is related to the strongest downstream baroclinicity in $\mathrm{fc} 17 \_12$. In fc17_00, where the temporal decrease of the low-level temperature gradient is strongest, the DRW is weakest and its track too far south. In all cases, baroclinicity increases again near the end of the propagation phase, which might be associated with warm frontogenesis.

According to the general concept of DRWs, they receive a large proportion of their energy from the

\footnotetext{
${ }^{4}$ Moore and Montgomery (2005) hypothesized that low-level baroclinicity and moisture are the key parameters determining the intensity of DRWs during propagation.
}

continuous condensation of water vapor [e.g., Moore and Montgomery (2005), their Fig. 8]. The required moisture is presumably advected from the south. Here, the available low-level moisture is calculated as the specific humidity averaged vertically between 1000 and $700 \mathrm{hPa}$ and horizontally in the dark blue box shown in Fig. 2d, which is located to the south and southeast of the DRW. Backward trajectory calculations from the region of intense latent heating (not shown) confirmed that the predominant moisture advection into the DRW passes through this box. Stronger systems have a more intense circulation and are able to feed themselves with more moisture than weaker ones [as already found by Trier and Davis (2002) for mesoscale convective vortices]. It is expected that a reduction of the available low-level moisture results in a decreased intensity of the DRW. (If this reduction went below a critical threshold the DRW propagation mechanism might even come to an end.) Figure 10b shows that available low-level moisture decreases during the DRW propagation in all forecasts and the analyses. This is due on the one hand to the DRWs moving away from the most pronounced subtropical moisture reservoir in the western North Atlantic (see Fig. 3), and on the other hand to the reduced moisture advection into the dark blue box associated with the weakening DRWs. The strongest DRW in fc17_12 is the one with the largest values of available low-level moisture. The weakest DRW in forecast fc17_00 features a strong simultaneous decrease of low-level moisture and low-level PV at 1800 UTC 18 December (cf. Figs. 9a and 10b).

In summary, during propagation the weakest DRW in fc17_00 is characterized by the smallest low-level baroclinicity downstream and the strongest reduction of available low-level moisture during propagation. In contrast, the strongest DRW in fc17_12 is related to the most 

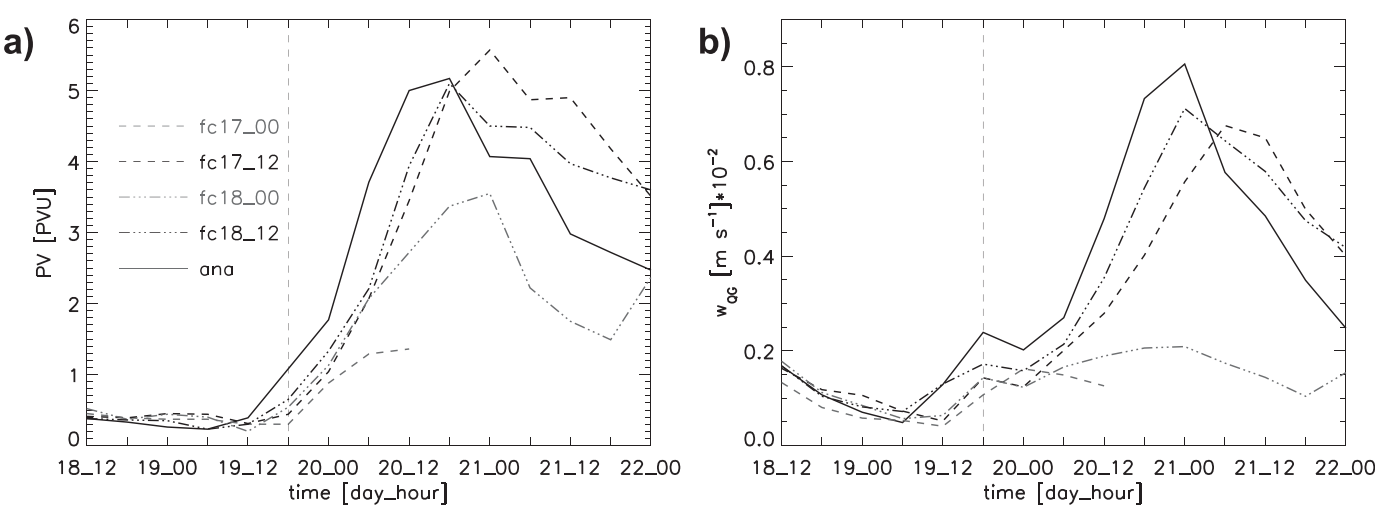

FIG. 11. Time development of (a) the upstream positive PV amount at $250 \mathrm{hPa}$ (averaged over the black box in Fig. 2d, PVU) and (b) the upper-level-induced QG vertical velocity at $700 \mathrm{hPa}$ (averaged over the gray box in Fig. 2d, $10^{-2} \mathrm{~m} \mathrm{~s}^{-1}$ ) for the forecasts and the analysis for the time period 1200 UTC 18 Dec-0000 UTC 22 Dec 2005. The dashed-dotted line separates the propagation from the intensification phase.

intense baroclinicity and the largest moisture availability. This supports the hypothesis that the temperature gradient downstream and the low-level moisture to the south of the DRW track are crucial factors determining the amplitude of the DRW during propagation.

\section{3) IMPACT FROM THE UPPER TROPOSPHERE DURING THE DRW INTENSIFICATION}

To identify the reason for the variability of the DRW intensification in the forecasts, the amplitude of the upper-level PV anomalies and of the vertical velocity field induced by these anomalies in the midtroposphere will be investigated. Whereas the distance to the upperlevel jet (see above) is a purely geometric measure of the potential impact from the tropopause level, averaging the $\mathrm{PV}$ on $250 \mathrm{hPa}$ within the black box upstream of the position of the DRW (see Fig. 2d) provides a more dynamic measure of the upper-level forcing. However, this first-order measure does not account for the PV anomaly's peak intensity, structure, and exact location that can vary considerably as shown in Fig. 8. During the propagation of the DRW the upstream upper-level PV values are tropospheric (about 0.5 PVU, see Fig. 11a), consistent with the earlier result that DRW propagation is not (significantly) influenced by upper-level forcing. At the beginning of the intensification phase, upperlevel PV values increase in the analyses and all forecasts. However, the increase is too weak in the nonintensifying forecasts fc17_00 and fc18_00, indicating that the upstream upper-level PV anomaly is either too weak or too far away from the DRW in the forecasts that miss the rapid cyclone intensification.

To further substantiate this important point, the final parameter considered is the upper-level induced quasigeostrophic vertical velocity at $700 \mathrm{hPa}$ downstream of the DRW (averaged in the gray box in Fig. 2d). In this region, the low-level induced ascent is strong during the entire life cycle of the DRW. The hypothesis is that intensification (i.e., the strong decrease of central SLP) occurs if the regions with strong upper- and lower-levelinduced ascent start to overlap. The upper-level-induced ascent at $700 \mathrm{hPa}$ in the gray box decreases during the DRW propagation due to the retreating trough that instigated the formation of the MCV (Fig. 11b). At 0600 UTC 20 December the forecasts split into two categories, characterized either by a strong increase of the upper-level induced ascent or by a fairly constant upper-level forcing for ascent. The latter occurs for the forecasts that miss the rapid cyclone intensification. Therefore, in the poor forecasts the DRWs are not directly affected by the upper-level induced ascent due to the approaching upper-level trough, which turns out to be essential for the system's rapid intensification.

The remainder of this section is devoted to a closer investigation of the poor forecasts fc17_00 and fc18_00, which failed in producing the DRW intensification (Figs. $8 \mathrm{a}, \mathrm{c})$. For the earlier forecast there are two factors that might be responsible for the weak development: (i) the DRW itself is too weak at the beginning of the intensification period for the reasons outlined above, and (ii) the upper-level trough approaching from upstream appears to be too weak (Fig. 11a). For the later of the poor forecasts, the situation is more involved. This forecast produced a DRW with a correct intensity until at least 0000 UTC 20 December (Fig. 9a) that experienced a fairly correct approach of the upper-level PV anomaly until 0600 UTC 20 December (Fig. 11a). Although this seems to imply favorable conditions for intensification, the upper-level forcing for ascent does not increase (Fig. 11b) in agreement with the missing low-level amplification (Figs. 9a,b). 

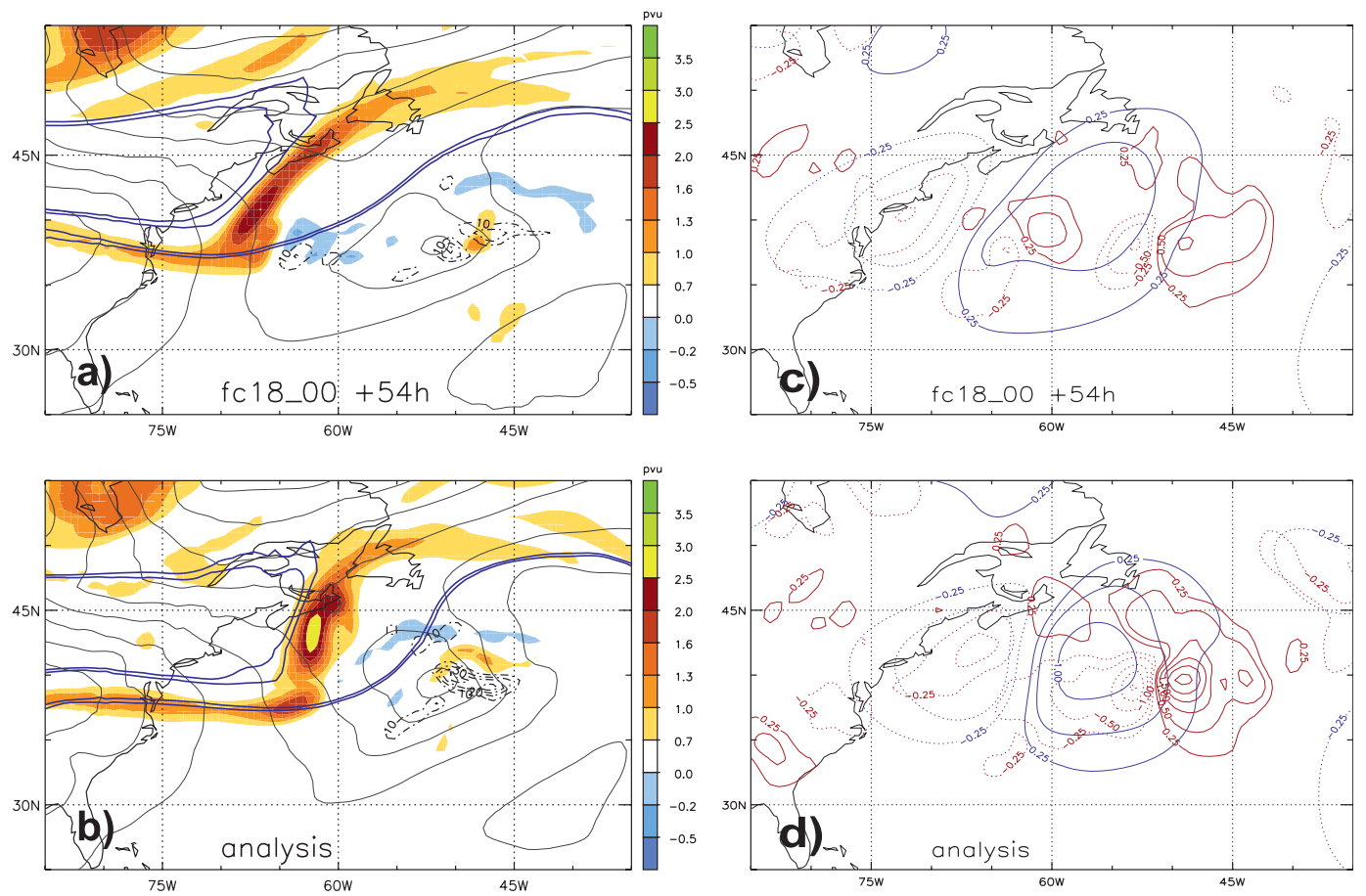

FIG. 12. (a),(b) PV at $500 \mathrm{hPa}$ (colors, PVU), SLP contours (hPa), latent heat release at $800 \mathrm{hPa}$ [black dashed lines, interval $10 \mathrm{~K}(6 \mathrm{~h})^{-1}$ ], and PV (2 PVU at $315 \mathrm{~K}$ blue). (c),(d) As in Fig. 5a, but at $0600 \mathrm{UTC} 20 \mathrm{Dec} 2005$.

Figure 12a shows that at 0600 UTC 20 December the approaching trough in fc18_00 has a leading edge near $67^{\circ} \mathrm{W}$ (see blue contours), that is, about $15^{\circ}$ to the west of the DRW (indicated by the SLP minimum), and that stratospheric air is penetrating below $500 \mathrm{hPa}$ (shown in colors). In contrast, in the analysis the leading edge of the trough is oriented more meridionally and the PV values are more pronounced at $500 \mathrm{hPa}$ (Fig. 12b). The stratospheric disturbance is closer to the DRW with a distance of less than $10^{\circ}$ between the deep stratospheric intrusion and the DRW. As a consequence, the trough in the forecast induces intense vertical motion mainly close to its leading edge (Fig. 12c), which produces latent heating (Fig. 12a) and in turn generates a new lowlevel positive PV anomaly about $10^{\circ}$ upstream of the DRW (shown 18 h later in Fig. 8c). Figure 12c indicates the low-level-induced ascent associated with this newly formed $\mathrm{PV}$ vortex at $39^{\circ} \mathrm{N}, 62^{\circ} \mathrm{W}$. The trough in the analysis, however, forces ascent in a large region that already encloses the location of the DRW (Fig. 12d). This indicates that vertical interaction in terms of collocated vertical motion forcing from the DRW and the upperlevel trough already started at 0600 UTC 20 December in the scenarios that contain the rapid intensification-but not in the others. For the forecast fc18_00, the different structure of the approaching upper-level trough, which does not support upper-level forcing of ascent in the region of the preexisting DRW precursor, appears as the main reason for the failed intensification.

Note that the importance of a low-level PV vortex interacting with an approaching upper-level trough for the subsequent evolution of the system is conceptually similar to the reintensification of a tropical cyclone when it interacts with midlatitude Rossby waves. In both situations there is a strong sensitivity to the structure, amplitude, and relative phase of the low-level and upperlevel features. For tropical cyclones, Ritchie and Elsberry (2007) found the systems' positions and the timing to be the important parameters for a successful intensification. Furthermore, it was hypothesized that the size and strength of the PV anomalies could have an impact on the development (Klein et al. 2002; Ritchie and Elsberry 2003).

\section{Summary and conclusions}

To shed further light on the phenomenon of diabatic Rossby waves, the life cycle of a particular DRW with three development phases over the North Atlantic has been investigated in operational analyses and forecast data from the ECMWF. The SLP minimum associated with the DRW could be tracked in ECMWF analyses from its generation as a $\mathrm{MCV}$, during the propagation as a DRW and the explosive intensification leading to 
a mature extratropical cyclone. By means of operational forecasts the environmental conditions have been examined that are crucial for (i) the strength of the DRW during the propagation and (ii) the eventual rapid intensification.

For this particular case, the diabatic heating associated with an MCV in the Gulf of Mexico has been identified as the mechanism to generate the DRW. This pathway is not necessarily representative for generating DRWs in other situations and further work will be required to obtain a more complete picture of the DRW generation mechanisms. With the aid of a quasigeostrophic omega diagnostic, the impact of the upper-level dynamics has been examined during the three distinct phases of the DRW evolution. For the MCV, upper- and lower-level-induced ascent at $700 \mathrm{hPa}$ overlapped, which has been interpreted as the upper-level forcing playing an important role for the genesis and maintenance of the MCV. As the system moved from the Gulf of Mexico into the North Atlantic its character changed and it continued to propagate as a DRW. The low-level PV anomaly was located over a strong baroclinic zone. The circulation induced by the anomaly enabled strong warm advection downstream leading to moist-diabatic processes and continuous PV generation. This corresponds to the theoretically found growth mechanism of a DRW (Raymond and Jiang 1990; Snyder and Lindzen 1991; Parker and Thorpe 1995). During this propagation phase no significant upper-level forcing has been found in the vicinity of the storm. This should be regarded as one of the key results of this study in that it clarifies that DRW propagation can occur without an upper-level forcing [as previously hypothesized by Wernli et al. (2002)]. The transition of the DRW into an intensifying cyclone was triggered by the superposition of upper- and lower-level-induced upward motion. The interaction of an approaching upper-level trough with the preexisting low-level vortex of the DRW resulted in an explosive pressure deepening of $34 \mathrm{hPa}$ in $24 \mathrm{~h}$.

The forecast performance for this DRW event has been investigated for a set of four operational ECMWF forecasts. All forecasts captured the genesis and propagation of the DRW. However, only two forecasts captured the system's explosive intensification whereas the other two forecasts missed the transition into a potentially damaging extratropical storm. A specially designed tracking algorithm has been used to quantify the characteristics of the DRW itself and of the environmental conditions during propagation and intensification. The amplitude of the low-level PV was tracked as an intensity measure of the system during propagation, which revealed a continuous weakening of about $0.4 \mathrm{PVU}$ in $30 \mathrm{~h}$. The identified main reasons for this weakening are the decrease along the track of both the low-level baroclinicity downstream of the DRW and of low-level moisture to the south of the system. This diagnostic analysis reveals that the strongest DRW occurred at the end of the propagation phase in the forecast where the decrease of these two key environmental parameters has been weakest. In contrast, the weakest DRW appeared in the forecast characterized by the strongest decrease of low-level baroclinicity and moisture.

After about $30 \mathrm{~h}$ of propagation, the amplitude of the low-level PV increased rapidly in the intensifying DRWs and remained nearly constant in the poor forecasts. To investigate the processes responsible for the intensification, the distance of the DRW to the jet at $250 \mathrm{hPa}$, the mean upstream PV at $250 \mathrm{hPa}$, and the quasigeostrophic upper-level forcing for ascent at $700 \mathrm{hPa}$ just downstream of the center of the DRW have been calculated. The intensification coincides with the approach of an upper-level trough as indicated by a reduced distance to the upper-level jet and increased upstream upper-level PV (Figs. 9e and 11a). In the poor forecasts the upperlevel impact is comparable to the intensifying systems until $12 \mathrm{~h}$ after the beginning of the intensification. Afterward a large difference appears in the upper-levelinduced ascent at $700 \mathrm{hPa}$ (Fig. 11b). In summary, the forecasts that missed the intensification reveal a too weak interaction of the low-level PV anomaly with the midlatitude upper-level trough. This interaction problem is similar to the possible reintensification of tropical cyclones when moving into midlatitudes (e.g., Jones et al. 2003). These scenarios share common characteristics and constitute formidable dynamical problems and major challenges to present-day forecasting systems.

This study corroborates the potential of DRWs to serve as precursors for explosive cyclone intensification and highlights the difficulties in forecasting their evolution. Clearly, further studies are required to confirm these results and to better elucidate the variety of extratropical cyclones' life cycles associated with DRWs. Studying these systems based upon operational ECMWF data suffers in part from the relatively coarse temporal (and maybe less so, spatial) resolution. This prompts investigating the evolution of DRWs with the aid of highresolution limited-area model simulations. Eventually, in order to assess the potential relevance of DRW-induced explosively deepening cyclones, a climatology of DRWs would be highly desirable. Such a climatology could reveal the preferred regions of DRWs, the mechanisms for their generation, as well as their frequency and seasonal cycle. The system-relative analysis of environmental conditions developed in this study could be applied to a climatology of DRWs in order to obtain further insight into the dynamics of these potentially high-impact extratropical weather systems. 
Acknowledgments. The German Research Foundation (DFG) is gratefully acknowledged for funding the research group PANDOWAE (FOR896) that enabled this work. The code for the quasigeostrophic vertical motion diagnostic was derived from routines in NDDIAG, a diagnostics package developed and supported by NCASCMS (U.K. National Centre for Atmospheric Science Computational Modelling Services). We thank Sue Gray (University of Reading) for making this package available to us. We are also grateful to Patricia Heckendorn for providing us with an initial version of the DRW tracking algorithm. Finally, we thank Ron McTaggartCowan and an anonymous reviewer for their constructive, in-depth reviews that helped to improve this paper.

\section{REFERENCES}

Berrisford, P., 1988: Potential vorticity in extratropical cyclones. Ph.D. thesis, University of Reading, $168 \mathrm{pp}$.

Bosart, L. F., 1981: The President's Day snowstorm of 18-19 February 1979: A subsynoptic-scale event. Mon. Wea. Rev., 109, 1542-1566.

Clough, S. A., C. S. A. Davitt, and A. J. Thorpe, 1996: Attribution concepts applied to the omega equation. Quart. J. Roy. Meteor. Soc., 122, 1943-1962.

Dacre, H. F., and S. L. Gray, 2009: The spatial distribution and evolution characteristics of North Atlantic cyclones. Mon. Wea. Rev., 137, 99-115.

Deveson, A. C., K. A. Browning, and T. D. Hewson, 2002: A classification of FASTEX cyclones using a height-attributable quasi-geostrophic vertical-motion diagnostic. Quart. J. Roy. Meteor. Soc., 128, 93-117.

Gyakum, J. R., 1983: On the evolution of the QE II storm. Part I: Synoptic aspects. Mon. Wea. Rev., 111, 1137-1155.

_ 1991: Meteorological precursors to the explosive intensification of the QE II storm. Mon. Wea. Rev., 119, 1105-1131.

— - P. J. Roebber, and T. A. Bullock, 1992: The role of antecedent surface vorticity development as a conditioning process in explosive cyclone intensification. Mon. Wea. Rev., 120, 1465 1489.

Jones, S. C., and Coauthors, 2003: The extratropical transition of tropical cyclones: Forecast challenges, current understanding, and future directions. Wea. Forecasting, 18, 1052-1092.
Kenzelmann, P., 2005: Dynamik und Klimatologie von diabatischen Rossby Wellen (Dynamics and climatology of diabatic Rossby waves). Diploma thesis, ETH Zurich, 90 pp.

Klein, P. M., P. A. Harr, and R. L. Elsberry, 2002: Extratropical transition of western North Pacific tropical cyclones: Midlatitude and tropical cyclone contributions to reintensification. Mon. Wea. Rev., 130, 2240-2259.

Knippertz, P., and H. Wernli, 2010: A Lagrangian climatology of tropical moisture exports to the Northern Hemispheric extratropics. J. Climate, 23, 987-1003.

Mallet, I., J.-P. Cammas, P. Mascart, and P. Bechtold, 1999: Effects of cloud diabatic heating on the early development of the FASTEX IOP17 cyclone. Quart. J. Roy. Meteor. Soc., 125, 3439-3467.

Moore, R. W., and M. T. Montgomery, 2004: Reexamining the dynamics of short-scale, diabatic Rossby waves and their role in midlatitude cyclogenesis. J. Atmos. Sci., 61, 754-768.

, and - 2005: Analysis of an idealized, three-dimensional diabatic Rossby vortex: A coherent structure of the moist baroclinic atmosphere. J. Atmos. Sci., 62, 2703-2725.

,-- , and H. C. Davies, 2008: The integral role of a diabatic Rossby vortex in a heavy snowfall event. Mon. Wea. Rev., 136, 1878-1897.

Parker, D. J., and A. J. Thorpe, 1995: Conditional convective heating in a baroclinic atmosphere: A model of convective frontogenesis. J. Atmos. Sci., 52, 1699-1711.

Raymond, D. J., and H. Jiang, 1990: A theory for long-lived mesoscale convective systems. J. Atmos. Sci., 47, 3067-3077.

Ritchie, E. A., and R. L. Elsberry, 2003: Simulations of the extratropical transition of tropical cyclones: Contributions by the midlatitude upper-level trough to reintensification. Mon. Wea. Rev., 131, 2112-2128.

— and - 2007: Simulations of the extratropical transition of tropical cyclones: Phasing between the upper-level trough and tropical cyclones. Mon. Wea. Rev., 135, 862-876.

Rossa, A. M., H. Wernli, and H. C. Davies, 2000: Growth and decay of an extra-tropical cyclone's PV-tower. Meteor. Atmos. Phys., 73, 139-156.

Snyder, C., and R. S. Lindzen, 1991: Quasigeostrophic wave-CISK in an unbounded baroclinic shear. J. Atmos. Sci., 48, 76-86.

Trier, S. B., and C. A. Davis, 2002: Influence of balanced motions on heavy precipitation within a long-lived convectively generated vortex. Mon. Wea. Rev., 130, 877-899.

Uccellini, L. W., 1986: The possible influence of upstream upperlevel baroclinic processes on the development of the QE II storm. Mon. Wea. Rev., 114, 1019-1027.

Wernli, H., S. Dirren, M. A. Liniger, and M. Zillig, 2002: Dynamical aspects of the life cycle of the winter storm "Lothar" (14-26 December 1999). Quart. J. Roy. Meteor. Soc., 128, 405-427. 